\title{
Comparative performance of current definitions of sarcopenia against the prospective incidence of falls among community-dwelling seniors age 65 and older
}

\author{
H. A. Bischoff-Ferrari ${ }^{1,2}$ - J. E. Orav ${ }^{3}$ J. A. Kanis $^{4} \cdot$ R. Rizzoli $^{5} \cdot$ M. Schlögl ${ }^{1,2}$. \\ H. B. Staehelin ${ }^{6}$ - W. C. Willett ${ }^{7}$ - B. Dawson-Hughes ${ }^{8}$
}

Received: 25 February 2015 / Accepted: 27 May 2015 / Published online: 12 June 2015

(C) International Osteoporosis Foundation and National Osteoporosis Foundation 2015

\begin{abstract}
Summary In this study, we compare the extent to which seven available definitions of sarcopenia and two related definitions predict the rate of falling. Our results suggest that the definitions of Baumgartner and Cruz-Jentoft best predict the rate of falls among sarcopenic versus non-sarcopenic communitydwelling seniors.

Introduction The purpose of the study is to compare the extent to which seven available definitions of sarcopenia and two related definitions predict the prospective rate of falling.

Methods We studied a cohort of 445 seniors (mean age 71 years, $45 \%$ men) living in the community who were followed with a detailed fall assessment for 3 years. For comparing the rate of falls in sarcopenic versus non-
\end{abstract}

H. A. Bischoff-Ferrari

Heike.Bischoff@usz.ch

1 Department of Geriatrics and Aging Research, University Hospital Zurich, Raemistrasse 101, 8091 Zurich, Switzerland

2 Centre on Aging and Mobility, University of Zurich, Zurich, Switzerland

3 Department of Biostatistics, Harvard School of Public Health, Boston, MA, USA

4 Centre for Metabolic Bone Diseases, University of Sheffield, Sheffield, UK

5 Service of Bone Diseases, Geneva University Hospitals and Faculty of Medicine, Geneva, Switzerland

6 Department of Geriatrics, University of Basel, Basel, Switzerland

7 Department of Nutrition, Harvard School of Public Health, Boston, MA, USA

8 USDA Human Nutrition Research Center on Aging, Tufts University, Boston, MA, USA sarcopenic individuals, we used multivariate Poisson regression analyses adjusting for gender and treatment (original intervention tested vitamin D plus calcium against placebo). Of the seven available definitions, three were based on low lean mass alone (Baumgartner, Delmonico 1 and 2) and four required both low muscle mass and decreased performance in a functional test (Fielding, Cruz-Jentoft, Morley, Muscaritoli). The two related definitions were based on low lean mass alone (Studenski 1) and low lean mass contributing to weakness (Studenski 2).

Results Among 445 participants, 231 fell, sustaining 514 falls over the 3-year follow-up. The prospective rate of falls in sarcopenic versus non-sarcopenic individuals was best predicted by the Baumgartner definition based on low lean mass alone $(\mathrm{RR}=1.54 ; 95 \% \mathrm{CI} 1.09-2.18)$ with $11 \%$ prevalence of sarcopenia and the Cruz-Jentoft definition based on low lean mass plus decreased functional performance $(\mathrm{RR}=1.82 ; 95 \%$ CI 1.24-2.69) with 7.1\% prevalence of sarcopenia. Consistently, fall rate was non-significantly higher in sarcopenic versus non-sarcopenic individuals based on the definitions of Delmonico 1, Fielding, and Morley.

Conclusion Among the definitions investigated, the Baumgartner definition and the Cruz-Jentoft definition had the highest validity for predicting the rate of falls.

Keywords Community-dwelling seniors $\cdot$ Comparative performance $\cdot$ Falls $\cdot$ Prevalence $\cdot$ Sarcopenia

\section{Introduction}

The European population is aging rapidly, and the number of seniors aged 65 and older is expected to increase from 25 to $40 \%$ by 2030 [1], as is the number of seniors with physical disability and resulting 
consequences, such as falls, fractures, and loss of autonomy [2-4]. This causes enormous challenges to the individual, health economy, and societies as a whole [5-11]. A condition that is considered central to the development of physical disability, frailty, and their consequences are sarcopenia $[4,5,9,10,12-23]$ and the loss of muscle mass and strength [20, 24].

The prevalence of sarcopenia has been reported to range between 5 and $13 \%$ in seniors aged 60 to 70 years, and 11 and $50 \%$ in seniors age 80 and above [16, 25]. Given the high prevalence of sarcopenia and its costly consequences, it imposes an enormous and rising economic burden. Based on the effect of sarcopenia on increasing physical disability risk in older persons, the direct healthcare cost attributable to sarcopenia in the USA in 2000 was estimated to be $\$ 18.5$ billion [5], and a $10 \%$ reduction in sarcopenia prevalence would result in savings of $\$ 1.1$ billion per year in US healthcare costs [5]. To date, however, the development of effective treatments for sarcopenia is hampered by regulatory and consensual obstacles on how to define and measure sarcopenia as a medical condition in the senior population $[9,14]$.

The underlying conceptual model of sarcopenia is that low skeletal mass leads to decreased muscle strength, and that decreased strength impairs physical function, which is thought to cause an increased risk of falls and to precede physical disability and frailty [26]. Seven operational definitions of sarcopenia [15-17, 21-23, 27] have been published, and two main concepts have been proposed, one that is based on low muscle mass alone [21-23] and the other that requires both low muscle mass and decreased performance in a functional test within a composite definition of sarcopenia [15-17, 27].

Falls in senior adults are accepted as a severe complication of sarcopenia $[15,16,26]$ and are frequent and costly events [28]. One in three adults aged 65 and one in two aged 80 years and older fall each year $[29,30]$. Falls cause moderate to severe injuries in 20 to $30 \%$ of cases, which result in functional impairment and increase the risk of nursing home admission and mortality [30, 31]. In the USA, the total direct medical costs of fall injuries for seniors 65 years and older was $\$ 30$ billion in 2012 [28]. By 2020, the annual direct and indirect cost of fall injuries is expected to reach $\$ 67.7$ billion [32].

While several definitions of sarcopenia have been proposed, they lack comparative performance testing against important endpoints such as falls. In this manuscript, we compared the extent to which available operational definitions of sarcopenia predict the rate of falls in 445 community-dwelling seniors age 65 years and older who were followed for 3 years. For the primary analyses, we focused on the rate of falls as each fall carries an important risk of injury, functional decline, and loss of autonomy [30, 31].

\section{Methods}

\section{Cohort}

The study was based in the original Boston STOP-IT cohort, which enrolled 445 healthy community-dwelling seniors age 65 and older, of whom 430 were White, 11 were Black, and 4 were Asian. All participants provided written informed consent, and the study protocol was approved by the Human Investigation Review Committee at Tufts University, Boston, USA. The original study design was a 3-year double-blind placebo-controlled trial originally designed and powered to study the effect of cholecalciferol-calcium on bone mineral density [33]. A secondary analysis addressed the effect of cholecalciferol-calcium on a person's risk of falling [34]. Of 445 subjects who were randomized, 389 attended the 3-year follow-up visit, and 318 were still receiving study medication at the 3-year follow-up visit.

The following exclusion criteria were applied: therapy consisting of bisphosphonate, calcitonin, estrogen, tamoxifen citrate, or testosterone in the past 6 months or fluoride in the past 2 years; those with a history of renal disease or renal stone in the past 5 years; and those with current cancer, hyperparathyroidism, dietary calcium intake exceeding $1500 \mathrm{mg}$ /day, or laboratory evidence of kidney or liver disease. Additional exclusion criteria have been published previously [33].

\section{Fall definition and ascertainment}

Participants were asked to send a postcard after every fall, which was then followed by a telephone call from a staff member to assess the circumstances of the fall. In addition, falls were ascertained at every 6-month follow-up visit. Falls were defined as "unintentionally coming to rest on the ground, floor, or other lower level [35]." For the primary analyses, we focus on the total number of falls reflecting the mean incidence at the individual level. As each fall carries an important risk of injury, functional decline, and loss of autonomy, this was our pre-defined preferred outcome as opposed to falling at least once $[30,31]$.

\section{Measurements}

Throughout the 3-year trial, subjects were invited to the study center every 6 months for a follow-up visit (six visits). Baseline body mass index was measured as weight in kilograms divided by the square of the height in meters at the study center. Baseline 25-hydroxyvitamin D (25-OHD) levels were measured by a competitive protein binding assay as described by Preece et al. [36]. Total body lean mass, total body fat mass, and appendicular muscle mass was measured by dual-energy X-ray absorptiometry with use of a DPX-L scanner (Lunar Radiation, Madison, WI). Scanner software versions 1.2 and 
1.3 were used for data acquisition and analysis. Grip strength of the dominant and nondominant arms was measured twice at each visit and the higher of the two measurements for each arm was recorded. Gait speed was assessed by recording the time required to walk 15 feet. Subjects were asked to walk the 15 feet at their normal pace.

\section{Seven available definitions of sarcopenia and two related definitions}

\section{Three definitions focused on low muscle mass by DEXA alone}

The first definition was proposed by Baumgartner et al. in 1998 [21] and is based on appendicular skeletal lean mass (ALM). According to this definition, individuals whose ALM divided by height squared $\left(\frac{\mathrm{ALM}(\mathrm{kg})}{\mathrm{Height}^{2}\left(\mathrm{~m}^{2}\right)}\right)$ is 2 or more standard deviations below sex-specific means of the Rosetta study [37] reference data set are defined as sarcopenic. The 1996 Rosetta Study included 284 participants (148 women and 136 men), White and African-American, average age 47.6 years [37]. Cutoffs for the definition based on data from Rosetta study reference data set are $\leq 7.26 \mathrm{~kg} / \mathrm{m}$ [2] for men and $\leq 5.45 \mathrm{~kg} / \mathrm{m}^{2}$ for women. This definition is further referred to as "Baumgartner."

The second definition was proposed by Delmonico et al. in 2007 and is calculated in the same way but the reference population and threshold for low appendicular lean mass is different. According to this definition, individuals whose ALM divided by height squared $\left(\frac{\mathrm{ALM}(\mathrm{kg})}{\mathrm{Height}^{2}\left(\mathrm{~m}^{2}\right)}\right)$ is below the 20th percentile of sex-specific distribution of the reference population in the Health ABC study [22] are defined sarcopenic. The 2000 baseline cohort of the Health ABC study included 1761 participants (992 men and 769 women), 25-44 years old [38]. Cutoffs for the definition based on data from the Health ABC study reference data set are $\leq 7.25 \mathrm{~kg} / \mathrm{m}^{2}$ for men and $\leq 5.67 \mathrm{~kg} / \mathrm{m}^{2}$ for women. This definition is further referred to as "Delmonico 1."

The third definition was proposed by Delmonico et al. in 2009 and uses the same reference population (Health ABC) as in Delmonico 1 but extends to gender-specific residuals that also include fat mass [22]. Linear models regressing ALM by height and total body fat mass were fit separately to the data from men and women. For men, the resulting model was $\operatorname{ALM}(\mathrm{kg})=-22.59+24.21 \times$ height $(\mathrm{m})+0.21 \times$ total fat $\operatorname{mass}(\mathrm{kg})$. For women, the resulting model was $\mathrm{AL}(\mathrm{kg})=-$ $13.21+14.76 \times$ height $(\mathrm{m})+0.23 \times$ total fat mass $(\mathrm{kg})$. These models, when used on the population under study, give predicted values for ALM for each individual. Then for each individual, a residual is calculated as a difference between observed and predicted ALM. A positive residual indicates that the person is relatively more muscular than predicted by the model and a negative residual suggests that this person is less muscular than predicted. People whose residuals fall below the 20th percentile of the sex-specific distribution are defined as "sarcopenic." This definition is further referred to as "Delmonico 2."

Four definitions focused on both low muscle mass by DEXA and decreased performance in a functional test such as slow gait speed or reduced grip strength

The fourth definition was proposed by Cruz-Jentoft et al. for the European Working Group on Sarcopenia in Older People in 2010 [15]. In addition to low ALM, the definition also requires decreased gait speed performance and/or decreased grip strength. Sarcopenia is defined by two conditions: (1) $\frac{\mathrm{ALM}}{\text { height }^{2}} \leq 7.26 \mathrm{~kg} / \mathrm{m}^{2}$ for men and $\leq 5.54 \mathrm{~kg} / \mathrm{m}^{2}$ for women and (2) gate speed $<0.8 \mathrm{~m} / \mathrm{s}$ and/or low grip strength $<30 \mathrm{~kg}$ for men and $<20 \mathrm{~kg}$ for women. As the authors do not provide a definite recommendation on the cutoff for low appendicular mass, we used the cutoffs proposed by Baumgartner based on the Rosetta study. This definition is further referred to as "Cruz-Jentoft."

The fifth definition was proposed by Fielding et al. for the International Working Group on Sarcopenia in 2011 [16]. In addition to low ALM using Health $A B C$ as the reference population, the definition also requires decreased gait speed performance. Sarcopenia is defined by two conditions: (1) $\frac{\text { ALM }}{\text { height }^{2}} \leq 7.23 \mathrm{~kg} / \mathrm{m}^{2}$ for men and $\leq 5.67 \mathrm{~kg} / \mathrm{m}^{2}$ for women and (2) gate speed $<1 \mathrm{~m} / \mathrm{s}$. This definition is further referred to as "Fielding."

The sixth definition was proposed by Morley et al. for the Society for Sarcopenia, Cachexia, and Wasting Disorders "Sarcopenia with limited mobility" in 2011 [17]. In addition to low ALM, the definition requires low gait speed or a distance less than $400 \mathrm{~m}$ on the 6-min walk. Sarcopenia is defined by two conditions: (1) $\frac{\text { ALM }}{\text { height }^{2}}$ of 2 standard deviations of more below the mean of healthy individuals between 20 and 30 years of age of the same ethnic group, using NHANES IV [39] as the reference population. The 2008 NHANES IV cohort includes 2402 participants (1035 men and 1367 women), 20-30 years old [39]. Sarcopenia is defined by two conditions: (1) appendicular muscle mass $\leq 6.81 \mathrm{~kg} / \mathrm{m}^{2}$ for men and $\leq 5.18 \mathrm{~kg} / \mathrm{m}^{2}$ for women and (2) gate speed $<1.0 \mathrm{~m} / \mathrm{s}$. This definition is further referred to as "Morley."

The seventh definition was proposed by Muscaritoli et al. for the Special Interest Groups "cachexia-anorexia in chronic wasting diseases" and "nutrition in geriatrics" in 2010 [27]. In addition to low lean mass, the definition also requires low gait speed. To define low muscle mass, like other authors, a $-2 \mathrm{SD}$ cutoff is proposed, however, related to total body skeletal lean mass (TLM) as opposed to appendicular lean mass. For gait speed they propose $<0.8 \mathrm{~m} / \mathrm{s}$ as a cutoff for "low gait speed." For the low lean mass cutoff, Muscaritoli et al. refer to a paper 
by Janssen et al. [40]. In order to standardize TLM measurements, Janssen et al. propose using a skeletal mass index (SMI): $\mathrm{SMI}=\frac{\mathrm{SM}}{\text { BodyMass }} \times 100$ Sarcopenia is defined by two conditions: (1) SMI $\leq 37 \%$ for men and SMI $\leq 28 \%$ for women and (2) gate speed $<0.8 \mathrm{~m} / \mathrm{s}$. This definition is further referred to as "Muscaritoli."

We also identified two recently published related definitions proposed by Studenski et al. for the Foundation for the National Institutes of Health Biomarkers Consortium Sarcopenia Project [41]. "Studenski 1" refers to low lean mass and "Studenski 2" refers to low lean mass contributing to weakness. To define low lean mass, the authors use ALM adjusted for body mass index (ALM $\mathrm{BMI})$ and weakness is defined based on grip strength. As a reference data set for the $\mathrm{ALM}_{\mathrm{BMI}}$ thresholds, the authors use a pooled data set of 26,625 community-dwelling older adults age 65 years and older (11,427 men and 15,198 women) from nine studies (the Study of Osteoporotic Fractures (SOF), the Osteoporotic Fractures in Men (MrOS) Study, the Framingham study, the UConn clinical trials, the Boston Puerto Rican Health Study, the Rancho Bernardo Study, the InChianti, Health $\mathrm{ABC}$, and the AGES-Reykjavik study). The Studenski 1 definition of low lean mass alone is defined by $\mathrm{ALM}_{\mathrm{BMI}}<$ 0.789 men and $\mathrm{ALM}_{\mathrm{BMI}}<0.512$ for women. The Studenski 2 definition of low lean mass contributing to weakness requires two conditions: (1) grip strength $<26 \mathrm{~kg}$ for men and $<16 \mathrm{~kg}$ for women and (2) $\mathrm{ALM}_{\mathrm{BMI}}<0.789$ men and $\mathrm{ALM}_{\mathrm{BMI}}<0.512$ for women.

\section{Statistics}

All analyses were based on 445 seniors enrolled in the Boston STOP-IT trial. Prevalence of sarcopenic individuals at baseline was defined according to the seven definitions as outlined above. We used Poisson regression to compare the rate of falls between individuals classified as sarcopenic versus individuals classified as non-sarcopenic during the 36-month follow-up, for each of the seven available definitions of sarcopenia and the two related definitions. Because the original study design was a clinical trial, the model included adjustment for treatment and gender for the full data set. Fully adjusted models also included age, BMI, and baseline serum $25(\mathrm{OH}) \mathrm{D}$ level. To account for the reduced exposure time among patients who died or withdrew from the study, we used an offset in the Poisson regression equal to the number of days in the study.

\section{We performed several sensitivity analyses}

In the first, we performed subgroup analyses for the rate of falls stratified by gender to test whether there is a differential pattern among men and women. For the second sensitivity analysis, we used a binary variable (fall yes versus no) instead of the rate of falls as the outcome variable. For this analysis, we used logistic models adjusting for the same set of covariates as the Poisson models of the primary analyses.

\section{Results}

Fifty-two percent of the subjects fell during the 3-year study ( $48.9 \%$ of men and $54.7 \%$ of women). The 213 fallers sustained a total of 514 falls: $45 \%$ had 1 fall, $24 \%$ had 2 falls, and $31 \%$ had $3+$ falls. Mean \pm SD baseline $25-\mathrm{OHD}$ levels were $26.6 \pm 12.7 \mathrm{ng} / \mathrm{mL}(66.4 \pm 31.7 \mathrm{nmol} / \mathrm{L})$ in women and $33.2 \pm 14.2 \mathrm{ng} / \mathrm{mL}(82.9 \pm 34.9)$ in men. Table 1 describes baseline characteristics of the study population.

\section{Prevalence of sarcopenia}

Across the seven available definitions of sarcopenia plus the two related definitions, the prevalence of sarcopenia varied between 2.5 and $27.2 \%$ among women, and 3.1 and $20.4 \%$ among men (Table 2). The overall pattern suggested that the composite definitions were more conservative in defining sarcopenia compared with definitions that used ALM alone. Only one composite definition, the one by Muscaritoli et al., provided a higher prevalence of sarcopenia based on total lean mass plus low gait speed identifying $20.4 \%$ of men and $26.2 \%$ of women as sarcopenic.

Among the three definitions that used low lean mass alone based on ALM, the prevalence of sarcopenia in men and women combined varied between $11 \%$ (Baumgartner) and $21.4 \%$ (Delmonico 2). Among the other four definitions (excluding Muscaritoli) that used low lean mass based on ALM in combination with decreased performance in a functional test, the prevalence of sarcopenia in men and women combined varied between 2.7 and $7.1 \%$.

Regarding prevalence by gender, all definitions except Delmonico 1 and 2 suggested a similar prevalence of sarcopenia among men and women.

\section{Comparative performance with respect to rate of falls}

The prospective rate of falls in sarcopenic versus nonsarcopenic individuals was best predicted by the Baumgartner definition based on low ALM ( $R R=1.54 ; 95 \%$ CI 1.09-2.18) and the Cruz-Jentoft composite definition of low ALM plus low gait speed or decreased grip strength $(\mathrm{RR}=1.82 ; 95 \% \mathrm{CI}$ 1.24-2.69). For all other sarcopenia definitions but Delmonico 2 and Muscaritoli, sarcopenic individuals had a non-significantly higher rate of falls compared with nonsarcopenic individuals (Fig. 1, Table 3). For the two related definitions Studenski 1 and 2, seniors with low lean mass had 
Table 1 Baseline characteristics of the study population

\begin{tabular}{lllcc}
\hline Mean, $\mathrm{SD}( \pm)$ & Men $(N=199)$ & Women $(N=246)$ & $p^{*}$ & Combined $(N=445)$ \\
\hline Age, years & $70.70(4.60)$ & $71.15(4.62)$ & 0.30 & $70.95(4.61)$ \\
Height, meter $(\mathrm{m})$ & $1.74(0.06)$ & $1.60(0.06)$ & $<0.0001$ & $1.66(0.10)$ \\
Total body weight, kilogram $(\mathrm{kg})$ & $81.85(11.89)$ & $68.05(12.46)$ & $<0.0001$ & $74.22(14.00)$ \\
Body mass index, $\mathrm{kg} / \mathrm{m}^{2}$ & $27.01(3.36)$ & $26.70(4.78)$ & 0.42 & $26.84(4.20)$ \\
Total body lean mass, $\mathrm{kg}$ & $54.85(5.63)$ & $37.63(4.41)$ & $<0.0001$ & $45.29(9.91)$ \\
Total body fat mass, kg & $23.00(7.81)$ & $27.16(9.00)$ & $<0.0001$ & $25.31(8.73)$ \\
Appendicular lean muscle mass, $\mathrm{kg}$ & $24.81(3.06)$ & $15.93(2.31)$ & $<0.0001$ & $19.88(5.16)$ \\
Appendicular lean muscle mass/height sq. $\left(\mathrm{kg} / \mathrm{m}^{2}\right)$ & $8.19(0.83)$ & $6.24(0.76)$ & $<0.0001$ & $7.11(1.25)$ \\
Appendicular lean muscle mass adjusted for BMI & $0.93(0.11)$ & $0.61(0.09)$ & $<0.0001$ & $0.75(0.19)$ \\
Grip strength in dominant hand, kgF & $35.67(6.50)$ & $19.46(4.79)$ & $<0.0001$ & $26.67(9.82)$ \\
Gait speed, m/s & $1.05(0.22)$ & $1.00(0.20)$ & 0.01 & $1.02(0.22)$ \\
\hline
\end{tabular}

*Based on two-sample $t$ test for continuous variables and on $\chi^{2}$ test for categorical variables

a neutral and those with low lean mass contributing to weakness had a non-significantly lower rate of falls.

\section{Comparative performance with respect to rate of falls-by gender}

In the sensitivity analysis for the prospective rate of falls by gender (Table 3), all definitions but Delmonico 2 and the two related definitions Studenski 1 and 2 indicated a higher rate of falls among affected men, although this was significant only for the Delmonico 2 definition. For women, a similar validity of an increased rate of falls among sarcopenic women was documented for the Baumgartner and the Cruz-Jentoft definitions. Notably, however, for Delmonico 2, Muscaritoli and Studenski $1+2$, the direction was inverted with a suggested lower rate of falls among affected women.

\section{Comparative performance with respect to odds of becoming a faller}

In the sensitivity analysis for the odds of becoming a faller (Table 3), the composite definitions tended to work best with
Table 2 Prevalence of sarcopenia according to the seven available definitions and two related definitions

\begin{tabular}{|c|c|c|c|c|c|c|c|}
\hline \multirow[t]{2}{*}{ Definition } & \multicolumn{2}{|c|}{$\operatorname{Men}(N=199)$} & \multicolumn{2}{|c|}{ Women $(N=246)$} & \multirow[t]{2}{*}{$p^{*}$} & \multicolumn{2}{|c|}{ Both genders $(N=445$} \\
\hline & Frequency & $\%$ & Frequency & $\%$ & & Frequency & $\%$ \\
\hline \multicolumn{8}{|c|}{ Definitions based on low muscle mass alone } \\
\hline Baumgartner $^{\mathrm{a}}$ & 24 & 12.2 & 25 & 10.2 & 0.54 & 49 & 11.0 \\
\hline Delmonico $1^{\mathrm{a}}$ & 22 & 11.2 & 53 & 21.5 & 0.005 & 75 & 16.9 \\
\hline Delmonico $2^{\mathrm{a}}$ & 28 & 14.2 & 67 & 27.2 & 0.001 & 95 & 21.4 \\
\hline Studenski $1^{\mathrm{a}}$ & 19 & 9.6 & 33 & 13.4 & 0.06 & 52 & 11.7 \\
\hline \multicolumn{8}{|c|}{ Composite definitions based on low muscle and decreased performance in a functional test } \\
\hline Cruz-Jentoft & 13 & 6.6 & 18 & 7.4 & 0.85 & 31 & 7.1 \\
\hline Fielding ${ }^{\mathrm{b}}$ & 7 & 3.6 & 15 & 6.2 & 0.27 & 22 & 5.0 \\
\hline Morley & 6 & 3.1 & 6 & 2.5 & 0.77 & 12 & 2.7 \\
\hline Muscaritoli & 40 & 20.4 & 64 & 26.2 & 0.18 & 104 & 23.6 \\
\hline Studenski 2 & 3 & 1.55 & 11 & 4.51 & 0.10 & 14 & 3.1 \\
\hline
\end{tabular}

All definitions refer to appendicular lean mass (ALM) for DEXA muscle mass assessment except Muscaritoli, which refers to total body lean mass (TLM)

* $p$ values are based on Fisher's exact test comparing prevalence's of sarcopenia between men and women for the seven available definitions of sarcopenia and the two related definitions for low lean mass alone (Studenski 1) and for low lean mass contributing to weakness (Studenski 2)

${ }^{a}$ Two patients had missing data on appendicular lean muscle mass, height, or total fat mass. Sample size for these definitions is $N=443$

${ }^{\mathrm{b}}$ In addition to the participants mentioned above three participants had missing gait speed data. Sample size for fielding definition: $N=440$ 


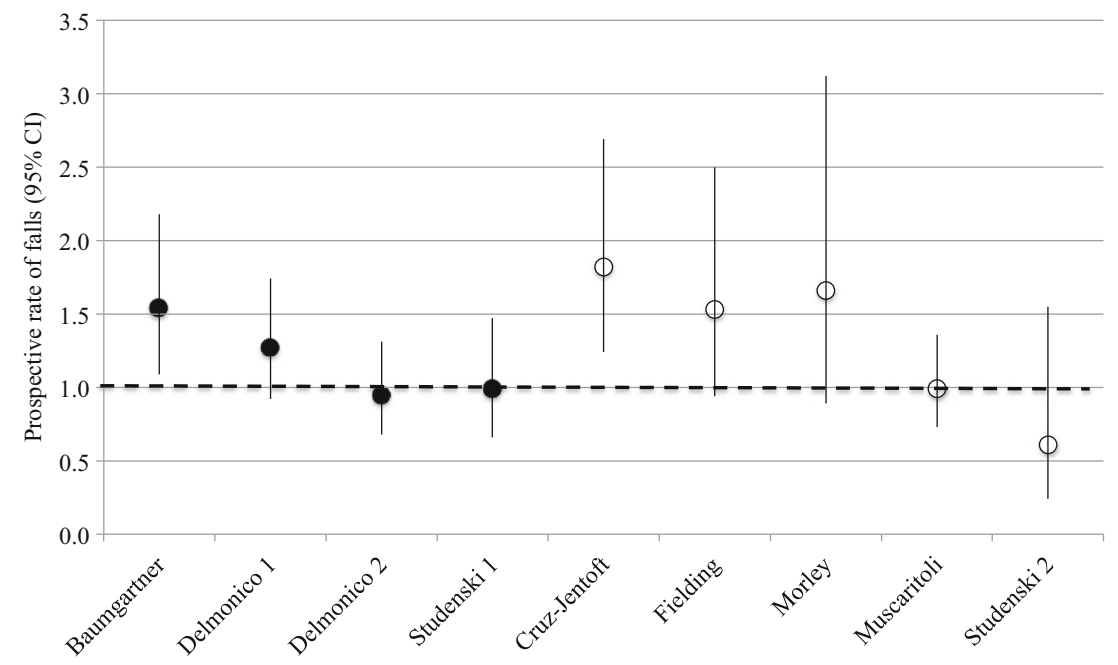

Fig. 1 Prospective rate of falls in sarcopenic versus non-sarcopenic seniors based on seven available definitions of sarcopenia plus two related definitions on low lean mass and low lean mass contributing to weakness. We used Poisson regression to compare the rate of falls between individuals classified as sarcopenic versus individuals classified as non-sarcopenic during the 3-year follow-up. The two related definitions by Studenski, refer to low lean mass (Studenski 1) and weakness due to low lean mass (Studenski 2). The filled circles represent definitions that were based on low lean mass alone, while the

sarcopenic individuals having higher odds of becoming a faller. Among the definitions that focused on low lean mass alone, the Baumgartner definition showed consistent validity. Also, consistent with the primary findings, the Delmonico 2, Muscaritoli, and Studenski $1+2$ definitions suggested non-significantly lower odds of becoming a faller among affected individuals. For the primary and sensitivity analyses, the additional adjustments by age, BMI, and baseline $25(\mathrm{OH}) \mathrm{D}$ status made no major difference in the results presented in Table 3.

\section{Discussion}

Falling is a widely accepted serious and clinically and economically relevant consequence of sarcopenia [15, 16]. To our knowledge, our study is the first attempt to define and compare the extent to which seven available definitions of sarcopenia [15-17, 21-23, 27] and two recently published related definitions [41] of low lean mass and low lean mass contributing to weakness predict the rate of falling. Our results, based on a cohort of 445 community-dwelling seniors age 65 years and older followed for 3 years, suggest that the definitions of Baumgartner referring to low appendicular lean mass alone and Cruz-Jentoft requiring both low appendicular lean mass and decreased performance in gait speed and/or grip strength best predict the rate of falls among sarcopenic versus non-sarcopenic community-dwelling seniors. open circles represent definitions that required both low lean mass and decreased functional performance. The analyses adjusted for treatment and gender. Best performance was documented for the Baumgartner and the Cruz-Jentoft definition of sarcopenia. The Delmonico 2 (adding body fat), the Muscaritoli (using total lean mass as oppose to ALM plus low gait speed) and the Studenski 1 (adjusting ALM for BMI) and Studenski 2 (low ALM adjusted for BMI contributing to weakness) definitions may not be useful in predicting fall incidence in communitydwelling seniors

With the same cutoff for low appendicular lean mass, the additional requirement of decreased function in the CruzJentoft definition increased the prediction of the rate of falls among sarcopenic individuals from an odds ratio of 1.54 (Baumgartner) to 1.82 (Cruz-Jentoft) and reduced the respective prevalence of sarcopenia from $11 \%$ (Baumgartner) to $7.1 \%$ (Cruz-Jentoft).

At the beginning of its conceptualization, the definition of sarcopenia was related to loss of muscle mass alone [24]. Then clinical observations in ageing cohorts emerged suggesting that muscle mass loss alone may not predict future strength decline [4, 42]. These important observations supported the rationale for the use of composite definitions of sarcopenia, which required both low muscle mass and the presence of decreased strength/gait performance to define sarcopenia [13, 14]. However, with the composite sarcopenia definition that purportedly captures decreased muscle mass and quality, several aspects may need to be considered. First, as decreased function is an integral part of these definitions, a better prediction of functional decline with the composite definition of sarcopenia as compared with low lean mass alone may be considered flawed. Second, by making it necessary that both criteria of low lean mass and decreased functional performance are met, most likely a progressed state of sarcopenia is identified, and the opportunity of early diagnosis of sarcopenia may be missed. Third, the requirement of both low lean mass and decreased performance in a functional test identifies very few community-dwelling seniors as sarcopenic [43]. 
Table 3 Comparative performance of the seven available sarcopenia definitions and two related definitions on low lean mass and low lean mass contributing to weakness with respect to rate of falls and odds of falling over the 3-year follow-up

\begin{tabular}{|c|c|c|}
\hline Sarcopenia definition & $\begin{array}{l}\text { Rate of falls sarcopenic } \\
\text { versus non-sarcopenic seniors }\end{array}$ & $\begin{array}{l}\text { Odds of falling sarcopenic } \\
\text { versus non-sarcopenic seniors }\end{array}$ \\
\hline \multicolumn{3}{|c|}{ Definitions based on low muscle mass alone } \\
\hline Baumgartner all & $1.54(1.09-2.18)$ & $1.27(0.70-2.32)$ \\
\hline Men & $1.10(0.60-2.02)$ & $1.85(0.77-4.45)$ \\
\hline Women & $1.97(1.32-2.94)$ & $0.93(0.40-2.13)$ \\
\hline Delmonico 1 all & $1.27(0.92-1.74)$ & $1.06(0.64-1.74)$ \\
\hline Men & $1.03(0.54-1.96)$ & $1.57(0.63-3.85)$ \\
\hline Women & $1.34(0.93-1.93)$ & $0.83(0.45-1.54)$ \\
\hline Delmonico 2 all & $0.95(0.68-1.31)$ & $0.75(0.47-1.18)$ \\
\hline Men & $1.83(1.13-2.96)$ & $2.05(0.90-4.71)$ \\
\hline Women & $0.59(0.38-0.92)$ & $0.42(0.23-0.74)$ \\
\hline Studenski 1 all & $0.99(0.66-1.47)$ & $1.18(0.66-2.12)$ \\
\hline Men & $0.97(0.49-1.92)$ & $1.47(0.56-3.83)$ \\
\hline Women & $0.99(0.60-1.62)$ & $1.00(0.47-2.10)$ \\
\hline \multicolumn{3}{|c|}{ Composite definitions based on low muscle and decreased performance in a functional test } \\
\hline Cruz-Jentoft all & $1.82(1.24-2.69)$ & $2.07(0.95-4.51)$ \\
\hline Men & $1.31(0.63-2.74)$ & $3.76(1.00-14.12)$ \\
\hline Women & $2.18(1.40-3.40)$ & $1.41(0.53-3.81)$ \\
\hline Fielding all & $1.53(0.94-2.50)$ & $1.67(0.69-4.08)$ \\
\hline Men & $1.24(0.47-3.27)$ & $6.60(0.78-55.92)$ \\
\hline Women & $1.64(0.93-2.87)$ & $0.99(0.34-2.83)$ \\
\hline Morley all & $1.66(0.89-3.12)$ & $0.93(0.29-2.93)$ \\
\hline Men & $1.15(0.39-3.35)$ & $1.04(0.21-5.30)$ \\
\hline Women & $2.32(1.08-5.01)$ & $0.85(0.17-4.33)$ \\
\hline Muscaritoli all & $0.99(0.73-1.36)$ & $0.91(0.59-1.41)$ \\
\hline Men & $1.14(0.69-1.88)$ & $1.36(0.67-2.74)$ \\
\hline Women & $0.89(0.60-1.32)$ & $0.70(0.39-1.25)$ \\
\hline Studenski 2 all & $0.61(0.24-1.55)$ & $0.70(0.24-2.06)$ \\
\hline Men & $0.82(0.14-4.67)$ & $0.52(0.05-5.83)$ \\
\hline Women & $0.53(0.18-1.59)$ & $0.71(0.20-2.40)$ \\
\hline
\end{tabular}

Italicized results indicate that the $95 \% \mathrm{CI}$ excludes 1 and thereby indicates significance
Alternatively, defining sarcopenia by low lean mass alone may be worth re-visiting for the following arguments: First, with low muscle mass alone, as proposed earlier [15, 44], the opportunity of capturing an early disease stage is available. Also, the introduction of multiple cutoffs to define the extend of muscle mass loss would provide the prospect of defining a spectrum of disease, such as pre-sarcopenia and sarcopenia $[15,44]$, similar to the definition of osteopenia and osteoporosis based on DEXA bone mineral density [45]. Second, by defining sarcopenia based on DEXA muscle mass alone, inter-rater variability and the influence of the patient's motivation would be eliminated from the diagnosis of sarcopenia. It is conceivable that regulatory agencies prefer an operatorindependent and exact technology-based definition of sarcopenia. Third, based on our findings, a significant $54 \%$ increased rate of falls among sarcopenic individuals based on the Baumgartner definition of low muscle mass alone is clinically relevant, especially as falls have multifactorial causes. Fourth, we document a useful prevalence of sarcopenia based on the Baumgartner sarcopenia definition of low muscle mass alone (11\%), while most composite definitions such as Cruz-Jentoft (7.1\%) and Fielding (5\%) defined a much lower prevalence likely due to the progressed disease stage. Finally, functional performance testing will require standardization efforts and additional resources in the assessment setup. Performance-based definitions of sarcopenia without muscle mass (i.e., the lowest $20 \%$ of the distribution for hand grip strength or lower limb strength) carry the same concerns $[43,46]$.

It is of interest that two available sarcopenia definitions and the two recently published related definitions of low lean mass and weakness due to low lean mass performed inconsistently compared to the other definitions. One is the Delmonico 2 definition, which defined a relatively high prevalence of 
sarcopenia among women with $27.2 \%$ and a significantly decreased rate of falls among sarcopenic versus nonsarcopenic women ( $\mathrm{RR}=0.59 ; 95 \%$ CI $0.38-0.92)$, while sarcopenic men according to the Delmonico 2 definition had a significantly increased rate of falls $(\mathrm{RR}=1.83 ; 95 \% \mathrm{CI}$ 1.13-2.96). This differential gender pattern was not seen for the other available definitions of low appendicular lean mass alone and questions the usefulness of this definition in clinical practice. An explanation may be the inclusion of fat mass in this Delmonico 2 definition, which may introduce a biased assessment among women.

Similarly, the Muscaritoli definition that required both low muscle mass and decreased gait speed performance defined a high prevalence of sarcopenia in both women $(20.4 \%)$ and men $(26.2 \%)$ if compared with the other available composite sarcopenia definitions which reported a prevalence of $7.4 \%$ or less among women and $6.6 \%$ or less among men. Also, the Muscaritoli definition showed poor performance in predicting the rate of falls with a non-significant $14 \%$ increased rate of falls among sarcopenic men and a non-significant $11 \%$ decreased rate of falls among sarcopenic women. A possible explanation of the limited performance of the Muscaritoli definition may be the fact that their muscle mass cutoffs referred to total muscle mass (TLM) as opposed to appendicular muscle mass (ALM) used by all other definitions. Finally, the most recent related definition by Studenski et al. [41] for the Foundation for the National Institutes of Health Biomarkers Consortium Sarcopenia Project identified both a very low prevalence of weakness due to low lean mass ( $4.5 \%$ among women and $1.6 \%$ among men) but also suggested a lower rate of falls among these individuals $(\mathrm{RR}=0.53$ among women and $\mathrm{RR}=$ 0.82 among men). Compared with the other seven available definitions of sarcopenia, the authors did not adjust appendicular muscle mass by height but by BMI. In addition, they required low grip strength to identify individuals with low muscle mass contributing to weakness as these individuals may benefit most from interventions. Despite their appealing concept, their sarcopenia-related definitions/thresholds of low lean muscle mass adjusted for BMI in combination with low grip strength did not predict the rate of falls in this study of community-dwelling seniors. While we agree with Studenski and colleagues that the reference population (Rosetta study [37]) of the Baumgartner definition may be too modest in size for optimal distributional characteristics for low lean mass, their alternative approach of using appendicular mass adjusted for BMI with a threshold assessed in a pooled data set from large cohort studies did not perform sufficiently well for fall prediction, both alone or in combination with weakness assessed by low grip strength.

The strength of this comparative performance exercise is that it is based on a cohort of 445 community-dwelling seniors followed for 3 years. Falls were ascertained comprehensively through postcards and at every 6-month follow-up visit. This is important because falls tend to be forgotten if no injuries are involved [47]. Also, the frequency of falls in this communitydwelling study sample is representative of what has been reported in the literature [48]. Additionally, the whole instrument library of sarcopenia (DXA lean mass, DXA fat mass, grip strength, gait speed, body mass index) was assessed in all participants at baseline allowing the comparative performance testing of the seven available definitions of sarcopenia published to date $[15-17,21-23,27]$ plus the two related definitions published recently [41] in a head-to-head manner.

There are also limitations to our study. Generalizability may be limited to community-dwelling older persons. Further, some may consider becoming a faller or not to be a more relevant or more conservative endpoint. Notably, however, as pointed out in the introduction of this paper, our preplanned primary endpoints was the total number of falls as each fall carries an important risk of injury, functional decline, and loss of autonomy [30, 31]. Further, our sensitivity analyses based on number of individuals who fell (odds of falling) support our main findings. Notably, however, falls may not be the sole outcome of relevance in sarcopenia. Alternative important endpoints of sarcopenia not tested in our study might be quality of life and loss of independence. Further, our results require validation in multiple cohorts. For the application of the individual definitions, two additional limitations need mentioning. First, for the sarcopenia definition proposed by Morley [17], the authors required low gait speed or a distance less than $400 \mathrm{~m}$ on the 6 -min walk next to the presence of low requested low ALM. As we did not have data on the 6-min walk test, we required all participants to have low gait speed. This may have been a more conservative approach than Morley et al. originally intended. Second, for the Crutz-Jentoft [15] definition, no defined threshold for ALM was selected by the authors and we chose the Baumgartner cutoff for optimal comparison with the Baumgartner definition. However, an alternative threshold mentioned by Cruz-Jentoft is the threshold for ALM by Newman et al. [49] referring to the sex-specific lowest $20 \%$ of the Health $\mathrm{ABC}$ cohort. In a sensitivity analysis (data not shown), we found that using this alternative ALM threshold for the Cruz-Jentoft definition did not improve but attenuate its prediction of falls among sarcopenic versus non-sarcopenic individuals. Finally, we acknowledge that some of the definitions will have low power due to the lower prevalence of sarcopenia.

In summary, our study is pertinent to the very timely efforts of consensus building on the definition of sarcopenia as a pivotal basis for the development of treatments against sarcopenia. Within this context, our comparative performance exercise of published definitions supports the possibility of a pragmatic approach that may be focused on low appendicular lean mass adjusted for body height alone as suggested by Baumgartner and colleagues. While, we acknowledge the somewhat enhanced prediction of fall incidence by the 
composite definition suggested by Cruz-Jentoft and colleagues, we point out that this is counterbalanced by the low percentage of individuals identified with sarcopenia and that these individuals likely represent a progressed disease stage and may therefore miss out on early treatment opportunities.

Funding sources The study was funded by the Baugarten Foundation and the International Foundation for the Promotion of Nutrition Research and Nutrition Education.

\section{Conflicts of interest None.}

\section{References}

1. Eberstadt N, Groth H. Europe's coming demographic challenge: unlocking the value of health. American Enterprise Institute for Health Policy Research. 2007

2. Book S. Alliance for aging research. http://www.agingresearch.org. 2013

3. Commission E. Healthy ageing: a keystone of a sustainable Europe. http://ec.europa.eu/health/ph_information/indicators/docs/healthy ageing_en.pdf. 2007

4. Visser M, Schaap LA (2011) Consequences of sarcopenia. Clin Geriatr Med 27(3):387-399

5. Janssen I, Shepard DS, Katzmarzyk PT, Roubenoff R (2004) The healthcare costs of sarcopenia in the United States. J Am Geriatr Soc 52(1):80-85

6. WHO. A glossary of terms for community health care and services. http://www.who.int/kobe_centre/ageing/ahp_vol5_glossary.pdf. 2004

7. CDC. The state of aging and health in America 2013. http://www. who.int/kobe centre/ageing/ahp vol5 glossary.pdf. 2013

8. Commission E. Healthy ageing: a key stone for a sustainable Europe. http://ec.europa.eu/health/archive/ph information/ indicators/docs/healthy_ageing_en.pdf. 2007

9. Motion Ai. Aging in motion: the facts about sarcopenia. http:// www.aginginmotion.org/wpcontent/uploads/2011/04/sarcopenia fact_sheet.pdf. 2013

10. Sayer AA (2010) Sarcopenia. BMJ 341:c4097

11. Evans WJ. Endpoints and indicators for the older population. http:// www.ema.europa.eu/docs/en_GB/document_library/Presentation/ 2012/04/WC500125114.pdf. 2012

12. Fried LP, Tangen CM, Walston J et al (2001) Frailty in older adults: evidence for a phenotype. J Gerontol A Biol Sci Med Sci 56(3): M146-M156

13. van Kan Abellan G, Andre E, Bischoff Ferrari HA (2009) Carla task force on sarcopenia: propositions for clinical trials. J Nutr Health Aging 13(8):700-707

14. Visser M (2009) Towards a definition of sarcopenia - results from epidemiologic studies. J Nutr Health Aging 13(8):713-716

15. Cruz-Jentoft AJ, Baeyens JP, Bauer JM et al (2010) Sarcopenia: European consensus on definition and diagnosis: report of the European Working Group on Sarcopenia in Older People. Age Ageing 39(4):412-423

16. Fielding RA, Vellas B, Evans WJ et al (2011) Sarcopenia: an undiagnosed condition in older adults. Current consensus definition: prevalence, etiology, and consequences. International working group on sarcopenia. J Am Med Dir Assoc 12(4):249-256

17. Morley JE, Abbatecola AM, Argiles JM et al (2011) Sarcopenia with limited mobility: an international consensus. J Am Med Dir Assoc 12(6):403-409
18. Vellas B, Pahor M, Manini T et al (2013) Designing pharmaceutical trials for sarcopenia in frail older adults: EU/US task force recommendations. J Nutr Health Aging 17(7):612-618

19. Studenski S (2009) What are the outcomes of treatment among patients with sarcopenia? J Nutr Health Aging 13(8):733-736

20. Morley JE, Baumgartner RN, Roubenoff R, Mayer J, Nair KS (2001) Sarcopenia. J Lab Clin Med 137(4):231-243

21. Baumgartner RN, Koehler KM, Gallagher D et al (1998) Epidemiology of sarcopenia among the elderly in New Mexico. Am J Epidemiol 147(8):755-763

22. Delmonico MJ, Harris TB, Lee JS et al (2007) Alternative definitions of sarcopenia, lower extremity performance, and functional impairment with aging in older men and women. J Am Geriatr Soc 55(5):769-774

23. Delmonico MJ, Harris TB, Visser M et al (2009) Longitudinal study of muscle strength, quality, and adipose tissue infiltration. Am J Clin Nutr 90(6):1579-1585

24. Rosenberg IH (1997) Sarcopenia: origins and clinical relevance. J Nutr 127(5 Suppl):990S-991S

25. von Haehling S, Morley JE, Anker SD (2010) An overview of sarcopenia: facts and numbers on prevalence and clinical impact. J Cachex Sarcopenia Muscle 1(2):129-133

26. Lang T, Streeper T, Cawthon P, Baldwin K, Taaffe DR, Harris TB (2010) Sarcopenia: etiology, clinical consequences, intervention, and assessment. Osteoporos Int 21(4):543-559

27. Muscaritoli M, Anker SD, Argiles J et al (2010) Consensus definition of sarcopenia, cachexia and pre-cachexia: joint document elaborated by Special Interest Groups (SIG) "cachexia-anorexia in chronic wasting diseases" and "nutrition in geriatrics". Clin Nutr 29(2):154-159

28. Stevens JA, Corso PS, Finkelstein EA, Miller TR (2006) The costs of fatal and nonfatal falls among older adults. Injur Prevent 12:290 295, 2006

29. Tromp AM, Pluijm SM, Smit JH, Deeg DJ, Bouter LM, Lips P (2001) Fall-risk screening test: a prospective study on predictors for falls in community-dwelling elderly. J Clin Epidemiol 54(8): $837-844$

30. Tinetti ME, Williams CS (1997) Falls, injuries due to falls, and the risk of admission to a nursing home. $\mathrm{N}$ Engl J Med 337(18):1279-1284

31. Sterling DA, O'Connor JA, Bonadies J (2001) Geriatric falls: injury severity is high and disproportionate to mechanism. J Trauma 50(1):116-119

32. Englander F, Hodson TJ, Terregrossa RA (1996) Economic dimensions of slip and fall injuries. J Forensic Sci 41(5):733-746

33. Dawson-Hughes B, Harris SS, Krall EA, Dallal GE (1997) Effect of calcium and vitamin $\mathrm{D}$ supplementation on bone density in men and women 65 years of age or older. N Engl J Med 337(10):670-676

34. Bischoff-Ferrari HA, Orav EJ, Dawson-Hughes B (2006) Effect of cholecalciferol plus calcium on falling in ambulatory older men and women: a 3-year randomized controlled trial. Arch Intern Med 166(4):424-430

35. Buchner DM, Hornbrook MC, Kutner NG et al (1993) Development of the common data base for the FICSIT trials. J Am Geriatr Soc 41(3):297-308

36. Preece MA, O'Riordan JL, Lawson DE, Kodicek E (1974) A competitive protein-binding assay for 25-hydroxycholecalciferol and 25-hydroxyergocalciferol in serum. Clin Chim Acta 54(2):235-242

37. Gallagher D, Visser M, De Meersman RE et al (1997) Appendicular skeletal muscle mass: effects of age, gender, and ethnicity. J Appl Physiol 83(1):229-239

38. Pichard C, Kyle UG, Bracco D, Slosman DO, Morabia A, Schutz Y (2000) Reference values of fat-free and fat masses by bioelectrical impedance analysis in 3393 healthy subjects. Nutrition (Burbank, Los Angeles County, Calif) 16(4):245-254 
39. Kelly TL, Wilson KE, Heymsfield SB (2009) Dual energy X-ray absorptiometry body composition reference values from NHANES. PLoS One 4(9):e7038

40. Janssen I, Heymsfield SB, Ross R (2002) Low relative skeletal muscle mass (sarcopenia) in older persons is associated with functional impairment and physical disability. J Am Geriatr Soc 50(5):889-896

41. Studenski SA, Peters KW, Alley DE et al (2014) The FNIH sarcopenia project: rationale, study description, conference recommendations, and final estimates. J Gerontol A Biol Sci Med Sci 69(5):547-558

42. Visser M, Deeg DJ, Lips P, Harris TB, Bouter LM (2000) Skeletal muscle mass and muscle strength in relation to lowerextremity performance in older men and women. J Am Geriatr Soc 48(4):381-386

43. Scott D, Hayes A, Sanders KM, Aitken D, Ebeling PR, Jones G (2014) Operational definitions of sarcopenia and their associations with 5-year changes in falls risk in community-dwelling middleaged and older adults. Osteoporos Int 25(1):187-193
44. Murphy RA, Ip EH, Zhang Q et al (2014) Transition to sarcopenia and determinants of transitions in older adults: a population-based study. J Gerontol A Biol Sci Med Sci 69(6):751-758

45. Kanis JA (1994) Assessment of fracture risk and its application to screening for postmenopausal osteoporosis: synopsis of a WHO report. WHO Stud Group Osteop Int 4(6):368-381

46. Correa-de-Araujo R, Hadley E (2014) Skeletal muscle function deficit: a new terminology to embrace the evolving concepts of sarcopenia and age-related muscle dysfunction. J Gerontol A Biol Sci Med Sci 69(5):591-594

47. Cummings SR, Nevitt MC, Kidd S (1988) Forgetting falls. The limited accuracy of recall of falls in the elderly. J Am Geriatr Soc 36(7):613-616

48. Bischoff-Ferrari HA (2011) The role of falls in fracture prediction. Curr Osteoporos Rep 9(3):116-121

49. Newman AB, Kupelian V, Visser M et al (2003) Sarcopenia: alternative definitions and associations with lower extremity function. $\mathrm{J}$ Am Geriatr Soc 51(11):1602-1609 